\title{
Radiation Ionizing, Diagnostic Imaging
}

National Cancer Institute

\section{Source}

National Cancer Institute. Radiation Ionizing, Diagnostic Imaging. NCI Thesaurus. Code C18980.

A technique for medical imaging that uses ionizing radiation like X-rays and gamma rays. Both $\mathrm{x}$-rays and gamma rays are high energy, short wavelength electromagnetic radiation that are capable of penetrating and passing through most tissues. The technique is highly useful to determine the presence or absence of a specific medical condition. 